This is corrected version of manuscript with changes made according to the Reviewers' comments and submitted to Journal of Luminescence. The final version is available here:

https://www.sciencedirect.com/science/article/pii/S0022231318314339

\title{
Impact of intrinsic defects on excitation dependent carrier lifetime in thick 4H-SiC studied by complementing microwave photoconductivity, free-carrier absorption and time-resolved photoluminescence techniques
}

\author{
Patrik Ščajev"1, , Saulius Miasojedovas1, Liudvikas Subačius², Kęstutis Jarašiūnas1, \\ Alexander V. Mazanik ${ }^{3}$, Olga V. Korolik ${ }^{3}$, Masashi Kato ${ }^{4,5}$ \\ ${ }^{1}$ Institute of Photonics and Nanotechnology, Vilnius University, Sauletekio Ave. 3, LT- \\ 10257 Vilnius, Lithuania \\ ${ }^{2}$ Optoelectronics Department, Center for Physical Sciences and Technology, Sauletekio \\ Ave. 3, LT-10257, Vilnius, Lithuania \\ ${ }^{3}$ Energy Physics Department, Belarusian State University, Nezalezhnastsi Ave. 4, Minsk \\ 220030, Belarus \\ ${ }^{4}$ Department of Engineering Physics, Electronics and Mechanics, Nagoya Institute of \\ Technology, Nagoya 466-8555, Japan \\ ${ }^{5}$ Frontier Research Institute for Materials Science, Nagoya Institute of Technology, Nagoya \\ 466-8555, Japan
}

\begin{abstract}
Photoexcited carrier dynamics in $10^{14}-10^{18} \mathrm{~cm}^{-3}$ density range was investigated by using complementary optical and microwave techniques. Bulk lifetime decrease from $4 \mu$ s to $460 \mathrm{~ns}$ with excitation and its increase with temperature were observed in bulk n-type $4 \mathrm{H}-\mathrm{SiC}$ crystal. The latter data and modeling of excitation-dependent hole capture with subsequent electron capture provided the trap position at $\mathrm{E}_{\mathrm{V}}+0.19 \mathrm{eV}$ and electron (hole) lifetimes of $360 \mathrm{~ns}$ (100 ns), correspondingly, at high excitation conditions. Lifetime increase with temperature was observed due to the trap thermal activation and reduction of electron capture cross section. The trap origin was discussed in terms of silicon vacancy related and titanium point defects. Photoluminescence spectra in 24-300 K range revealed bound exciton luminescence and nitrogen-aluminum donor-acceptor pair band, which excitation dependent and time-resolved dynamics was analyzed in wide temperature range.
\end{abstract}

Keywords: 4H-SiC, microwave photoconductivity, free carrier absorption, photoluminescence, carrier recombination, traps.

\footnotetext{
${ }^{1)}$ E-mail: patrik.scajev@ff.vu.lt
} 
This is corrected version of manuscript with changes made according to the Reviewers' comments and submitted to Journal of Luminescence. The final version is available here:

https://www.sciencedirect.com/science/article/pii/S0022231318314339

\section{Introduction}

There has been considerable interest in characterizing the lifetimes of carriers within epitaxial layers of $4 \mathrm{H}-\mathrm{SiC}$ [1-7] as minority carrier lifetime is a key parameter for SiC bipolar devices. However, wide variation in measured lifetimes stems from differences in experimental conditions, such as injection level, temperature, as well from impact of point and planar defects. Typically, in n-type $4 \mathrm{H}-\mathrm{SiC}$ main recombination mechanism is attributed to deep $\mathrm{Z}_{1 / 2}$ traps [1,5], providing an increase in lifetime with excitation and temperature. Growth at high $\mathrm{C} / \mathrm{Si}$ ratio leads to low $\mathrm{Z}_{1 / 2}$ trap concentration (below $10^{13} \mathrm{~cm}^{-3}$ ) [5]; in this kind crystals lifetime decreases by increasing the excitation intensity, pointing to recombination via other pathways. Similar effect of lifetime decrease with increase of nitrogen doping was observed in [7] and not correlated with low $Z_{1 / 2}$ trap density. Thus, when intrinsic $\mathrm{Z}_{1 / 2}$ trap density is low, other recombination mechanisms may come into play, such as dislocations and stacking faults [8-10] demonstrating the peculiar phenomena of either decreasing or increasing minority-carrier lifetime depending on the stacking fault related energy levels [11]. Nevertheless, point defect related recombination mechanisms cannot be neglected [2,12]. Hence, study of defect related mechanisms requires their accurate discrimination from the surface recombination impact by using thick crystals, combining various measurement techniques, and numerical carrier transport modelling.

Therefore, in this work we applied the microwave photoconductance decay (MPCD), freecarrier absorption (FCA), and time-resolved photoluminescence (TRPL) techniques for investigation of carrier lifetime features in bulk $120 \mu \mathrm{m}$ thick $4 \mathrm{H}-\mathrm{SiC}$ in wide excitation and temperature ranges. The MPCD technique has the best sensitivity but exhibits the saturating conductivity response at high excitations. The latter drawback can be corrected by calibration procedure of MPCD signal on excess carrier density [13]. After calibration, the MPCD transients in reflection and transmission become identical in wide excitation range, from $2 \times 10^{14}$ to $10^{18} \mathrm{~cm}^{-3}$. TRPL also exhibits high sensitivity but needs PL decay time correction for determination of carrier lifetime (i.e. requires knowledge of PL intensity dependence on excess carrier density), while FCA [14] is applicable only at high excited carrier densities (above $5 \times 10^{15} \mathrm{~cm}^{-3}$ ) due to relatively low absorption changes by excess free carriers.

The earlier study of this type but thinner 4H-SiC crystal [13] has shown decrease of carrier lifetime with increase of excess carrier density at rather low excitations due to surface recombination. Present work focuses on lifetime measurements in a thick $120 \mu \mathrm{m}$ epilayer, where surface recombination has smaller impact and defect-governed bulk lifetime dependences on 
This is corrected version of manuscript with changes made according to the Reviewers' comments and submitted to Journal of Luminescence. The final version is available here:

https://www.sciencedirect.com/science/article/pii/S0022231318314339

excitation and temperature can be investigated more thoroughly. The observed unusual ShockleyReed-Hall (SRH) lifetime decrease with excitation (from $4 \mu$ s to $0.5 \mu$ s in $10^{14}-10^{18} \mathrm{~cm}^{-3}$ excitation range) and its increase with temperature (by order of magnitude) was attributed to shallow intrinsic silicon vacancy related hole traps at $E_{V}+0.19 \mathrm{eV}$, which electrical activity depends on excited carrier density. We ascribe the observed lifetime decrease by defect-limited transition from minority to bipolar carrier lifetime. The lifetime temperature measurements confirmed this hypothesis.

\section{Sample and techniques}

A commercial $150 \mu \mathrm{m}$ thick n-type $4 \mathrm{H}-\mathrm{SiC}$ wafer grown on heavily-doped $4 \mathrm{H}-\mathrm{SiC}$ (0001) substrate was utilized. The substrate was removed by mechanical polishing and the subsequent chemical mechanical polishing [15]. In this way, sample of $d=120 \mu \mathrm{m}$ thickness was prepared. Electron density in the sample was $n_{0}=(10 \pm 2) \times 10^{14} \mathrm{~cm}^{-3}$ and compensating aluminum concentration was $(3.5 \pm 0.5) \times 10^{14} \mathrm{~cm}^{-3}$ as determined by SIMS. Growth was performed at carbon rich conditions $(\mathrm{C} / \mathrm{Si} \approx 1.2)$ providing low $\mathrm{Z}_{1 / 2}$ density, estimated below $5 \times 10^{12} \mathrm{~cm}^{-3}[16]$.

Sample excitation was performed by the third harmonic $(355 \mathrm{~nm})$ of Nd:YAG laser with 25 ps pulses. Excitation fluence $I_{0}$ was tuned in a wide range by rotation of a half wave plate positioned before Glan prism. The photogenerated carrier density near the sample surface can be estimated by relation $\Delta N^{*}=\alpha I_{0} / h v$, where $\alpha=200 \mathrm{~cm}^{-1}$ is the absorption of $4 \mathrm{H}-\mathrm{SiC}$ at $355 \mathrm{~nm}$ [17] (here $h v=3.49 \mathrm{eV}$ is the pump quantum energy and $I_{0}$ is the excitation fluence in the sample (in $\left.\mathrm{mJ} / \mathrm{cm}^{2}\right)$ ). For further analysis of instantaneous carrier lifetime dependence on excitation at different delay times, the statistically averaged carrier density $\Delta N(z c, t)$ was used, which is derived from condition $\int_{0}^{c c} \Delta N(z, t) d z=\int_{z c}^{l} \Delta N(z, t) d z[18]$.

The sample for MPCD measurements was mounted into the rectangular waveguide setup and illuminated via longitudinal slot of $2 \times 4 \mathrm{~mm}$ size by the Nd:YAG laser beam (for setup details, see [13]). For all given below measurements the illuminated surface area of the sample was of $1.6 \mathrm{x}$ $1.6 \mathrm{~mm}^{2}$. Complementary measurements by FCA technique provided carrier lifetimes at high excess carrier density (in $10^{16}-10^{18} \mathrm{~cm}^{-3}$ range), using the same excitation conditions $(355 \mathrm{~nm}$ ) and the first laser harmonics $(1064 \mathrm{~nm})$ for probing of FCA decay [3]. The differential transmittivity of the sample (DT), caused by FCA, increased linearly with excitation in $0.05-5 \mathrm{~mJ} / \mathrm{cm}^{2}$ excitation range (with 0.97 slope), thus enabling simple determination of carrier lifetime, $\tau_{R}$. Temperature measurements of carrier lifetime in $80-800 \mathrm{~K}$ range were also performed by FCA technique. 
This is corrected version of manuscript with changes made according to the Reviewers' comments and submitted to Journal of Luminescence. The final version is available here:

https://www.sciencedirect.com/science/article/pii/S0022231318314339

PL spectra and decay kinetics were measured for analysis of defect impact on recombination. For excitation, the tunable wavelength pulses from Orpheus (Light Conversion) optical parametric amplifier (OPA) were used. The OPA was pumped by $\sim 160$ fs pulses of PHAROS (Light Conversion) laser, operating at $10 \mathrm{kHz}$ repetition rate. Excitation-dependent TRPL measurements were carried out using $355 \mathrm{~nm}$ pump wavelength. Gaussian spot diameter was 305 $\mu \mathrm{m}$ at 1/e width thus minimizing impact of carrier in-plane diffusion. PL emission spectra were measured in back-scattering geometry using a Hamamatsu streak camera and an Acton monochromator [19]. Temperature dependent (24-290 K) PL spectra were measured using a Nanofinder HE confocal spectrometer (LOTIS TII, Belarus-Japan [20]). Spectral resolution was equal to $0.1 \mathrm{~nm}$. Spectral calibration was done using a built-in gas-discharge lamp providing an accuracy better than $0.1 \mathrm{~nm} .355 \mathrm{~nm}$ DPSS CW laser was used as an excitation source. The samples were excited with spot diameter of about $2 \mu \mathrm{m}$. However, due to carrier diffusion the spot diameter is greatly increased up to a value of diffusion length $L_{D}=\left(D \tau_{R}\right)^{1 / 2}=10-20 \mu \mathrm{m}$ (estimated using the determined values of lifetime $\sim 400 \mathrm{~ns}$ and $D \sim 4-10 \mathrm{~cm}^{2} / \mathrm{s}$ [21]). Therefore, average excited carrier density will be $\Delta N=\alpha(355) P_{0} \tau_{R} /\left(8 L_{D}^{2} h v\right)=\alpha(355) P_{0} /(8 D h v)$, where absorption coefficient $\alpha(355$ $\mathrm{nm})=100-200 \mathrm{~cm}^{-1}$ [21]. Thus used $P_{0}=4 \mathrm{~mW}$ excitation power will provide average carrier density of $\sim 4 \times 10^{16} \mathrm{~cm}^{-3}$.

\section{Experimental results and discussion}

\subsection{Microwave photoconductance and free carrier absorption decays}

In Fig. 1, the experimental kinetics of MPCD and FCA decay are shown. The measurements in wide excitation range reveal the fast and slow decay components, varying from $1 \mu$ s to $0.25 \mu$ s and from $4.5 \mu$ s to $2.3 \mu \mathrm{s}$, respectively. The previously reported a single exponential FCA decay in 160 $\mu \mathrm{m}$-thick $4 \mathrm{H}-\mathrm{SiC}$ (at $351 \mathrm{~nm}$ excitation) provided bulk lifetime of $800 \mathrm{~ns}$ in $10^{17}-5 \times 10^{17} \mathrm{~cm}^{-3}$ range, while the faster FCA component emerged only at excitations above $10^{18} \mathrm{~cm}^{-3}$ due to impact of nonlinear carrier recombination [3]. Similar measurements in $160 \mu \mathrm{m}$ thick n-type 4H-SiC epilayer with carrier concentration in the mid- $10^{14} \mathrm{~cm}^{-3}$ range [1] revealed injection-dependent increase of carrier lifetimes, in accordance with transition from minority to bipolar recombination regime through $Z_{1 / 2}$ traps. Therefore, the feature of decreasing with excitation bulk carrier lifetime at rather low injection levels is different from the reported cases and needs deeper analysis. Thus we carried out PL measurements to unveil presence of defects and their impact on recombination dynamics. 
This is corrected version of manuscript with changes made according to the Reviewers' comments and submitted to Journal of Luminescence. The final version is available here:

https://www.sciencedirect.com/science/article/pii/S0022231318314339
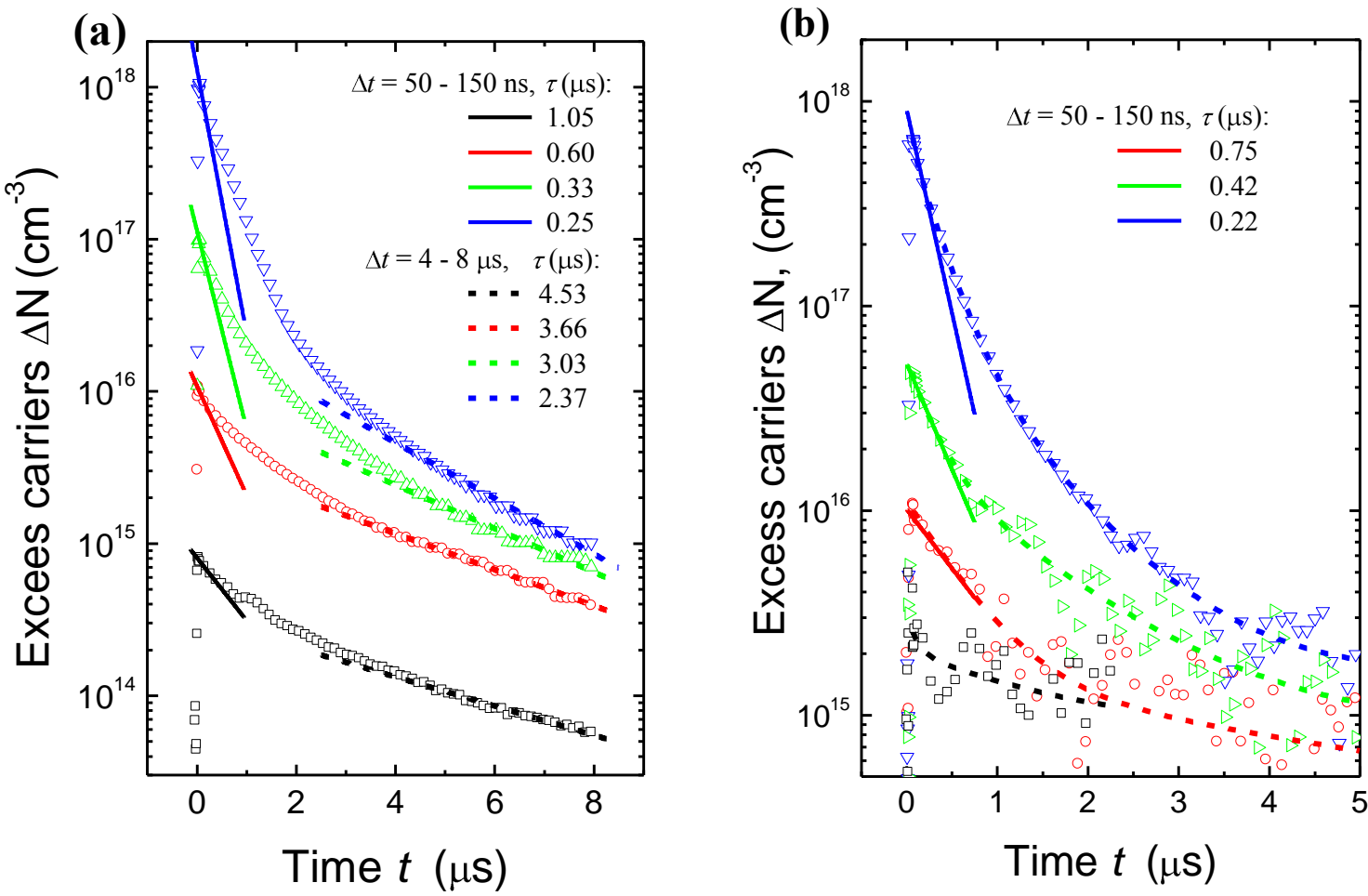

Fig. 1. Carrier density dependent decays measured by MPCD (a) and FCA (b) methods. Lines are linear fits in semilog scale. Fitted lifetime values $\tau_{\mathrm{R}}$ are given on the plots.

\subsection{Excitation dependent PL spectra and decays}

The excitation-dependent PL spectra and time-resolved PL decays provided information about origin of radiative recombination mechanisms and presence of traps in the sample. The PL spectra at room temperature show two main PL bands, B1 and B2, centered at $3.19 \mathrm{eV}$ and $2.96 \mathrm{eV}$, respectively (Figs. 2 and 3). Here, the B1 band corresponds to free carrier bandgap emission [8], while the B2 band points out to defect related emission being untypical for low C/Si ratio [2,23].

The temperature dependent PL spectra (Fig. 2) show six distinguishable features. Here BX, 1PB, 2PB correspond to bound exciton peaks, free exciton one- and two-phonon bands, respectively [24,25]. Nitrogen to hole trap donor-acceptor pair (DAP) emission at 1PB, 2PB corresponds to one and two phonon bands, respectively. At higher temperatures, $1 \mathrm{~PB}$ and $2 \mathrm{~PB}$ correspond to free carrier one- and two- phonon bands. BX ZPL phonon line observed at $3.25 \mathrm{eV}$ corresponds to excitonic bandgap of $E_{G X}=3.27 \mathrm{eV}$ [24]. Using exciton binding energy in $4 \mathrm{H}-\mathrm{SiC}$ of $\sim 20 \mathrm{meV}$ [26], bandgap of $3.29 \mathrm{eV}$ can be determined. Taking into account that our sample is grown at $\mathrm{C}$ rich conditions, nitrogen is dominantly positioned at hexagonal and cubic sites with activation energies 
This is corrected version of manuscript with changes made according to the Reviewers' comments and submitted to Journal of Luminescence. The final version is available here:

https://www.sciencedirect.com/science/article/pii/S0022231318314339

$E_{h}=61.4 \mathrm{meV}, E_{c}=125.5 \mathrm{meV}[5,25,27,28]$. Nitrogen at hexagonal site is dominant in DAP luminescence [29]. The photon energy corresponding to infinitely separated donor and acceptor (with zero Coulomb interaction) was estimated from the low-energy cut-off of the broad DAP peak, by using relation $E_{D A P}=E_{G}-E_{D}-E_{A}$ [30], to be $E_{D A P}=3.02 \mathrm{eV}$, latter provides the hole trap activation energy of $200 \pm 20 \mathrm{meV}$. Note, spectra shapes did not change with excitation in 1.2- 4 $\mathrm{mW}$ range, while the lateral and in-depth PL scans showed intensity variations only by few times (thus, indicating that DAP is not related to surface or structural defects).

At $\mathrm{T}>24 \mathrm{~K}$ bound exciton peaks are strongly quenched, whereas that of free carriers oneand two-phonon peaks are strongly enhanced jointly with DAP as free carriers concentration increases due to exciton ionization [21]. DAP band weakly changes on temperature, indicating that traps are not activated considerably. Nevertheless, the band-to-band and DAP 1PB peak differences remain similar as at low temperatures, providing $E_{D}+E_{A}=245 \mathrm{meV}$, leading to similar value of hole trap energy, $185 \pm 20 \mathrm{meV}$. Enlargement of bound exciton luminescence region (Fig. 2b) shows presence of peaks at $382.1 \mathrm{~nm}(3.2448 \mathrm{eV}), 381.7 \mathrm{~nm}(3.2482 \mathrm{eV})$, and $381.5 \mathrm{~nm}(3.2499$ $\mathrm{eV}$ ), which correspond to nitrogen on cubic site, aluminum on hexagonal site, and aluminum on cubic site, respectively [25]. This indicates that aluminum should be active in the defect B2 band luminescence. Indeed, Al activation energy of $200 \mathrm{meV}$ [25,31] well coincides with the determined one.
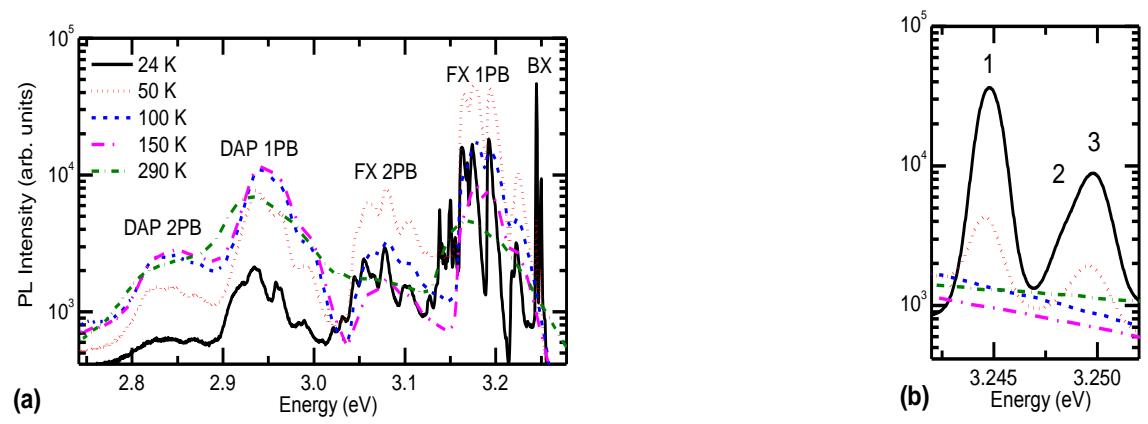

Fig. 2. (a) Temperature dependent PL spectra, recorded at $355 \mathrm{~nm}$ and $4 \mathrm{~mW}$ excitation. (b) Bound exciton peaks: 1 - nitrogen on cubic site, 2 - aluminum on hexagonal site, 3 - aluminum on cubic site. 
This is corrected version of manuscript with changes made according to the Reviewers' comments and submitted to Journal of Luminescence. The final version is available here:

https://www.sciencedirect.com/science/article/pii/S0022231318314339

Nearly quadratic increase of B1-band intensity $I_{P L}$ with excitation, $I_{P L} \sim B \Delta N^{1.92}$, was observed (Fig. 3b) due to dominant free carrier recombination at bandgap $I_{P L} \sim B \Delta N^{2}$ [32] as exciton binding energy is low in $\mathrm{SiC}(\sim 20 \mathrm{meV}[26])$. Therefore, $\tau_{R}=2 \tau_{P L}$ relation should hold for carrier lifetime determination, where $\tau_{P L}$ is the PL lifetime. The observed spectrally-integrated PL decay times in B1 band (within 3.06-3.31 eV, Fig. 4a) are thus twice shorter than those of FCA and MPCD decays (compare Fig. 1 and Fig. 4a). Moreover, the DAP PL emission kinetics in B2 band (integrated in 2.80-3.00 eV interval, Fig. 4b) has the delayed intensity peak due to the filling of aluminum traps.
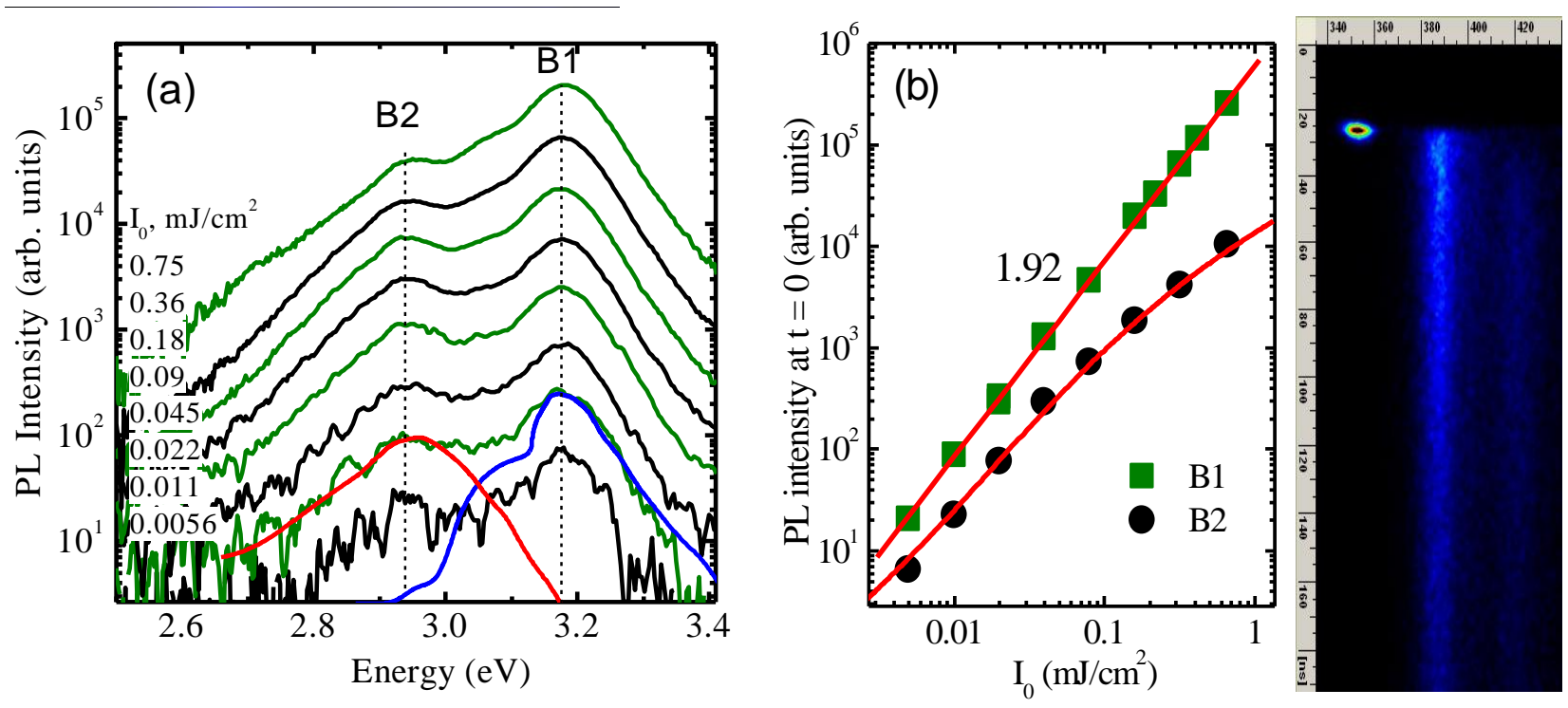

Fig. 3. (a) Time integrated (in $5 \mu$ s window) excitation dependent PL spectra at $300 \mathrm{~K}$. Two PL bands peaked at $3.19 \mathrm{eV}(389 \mathrm{~nm})$ and $2.96 \mathrm{eV}(419 \mathrm{~nm})$ were observed. (b) Excitation dependences of the bands TRPL decay (see Fig. 4) peak intensities. Line for B1 indicates linear fit, while curve for B2 is numerical fit (see details in the text). In (a) blue curve depicts band edge emission in low $\mathrm{C} / \mathrm{Si}$ ratio $4 \mathrm{H}-\mathrm{SiC}$ [23], while red one shows decomposed DAP impact. (c) PL trace in streak camera.

The excitation dependence of defect band B2 intensity can be calculated as $P L \sim N^{0} A l^{0}$. Here $N^{0}$ is the neutral nitrogen density, while $A l^{0}$ is the density of photo-neutralized aluminum traps with $E_{A l}$ activation energy. Assuming that recombination is slow as compared to capture, the capture dynamics to the aluminum and nitrogen impurities can be described by equation [33]: 
This is corrected version of manuscript with changes made according to the Reviewers' comments and submitted to Journal of Luminescence. The final version is available here:

https://www.sciencedirect.com/science/article/pii/S0022231318314339

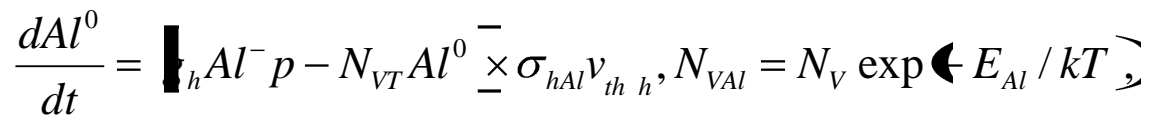

$$
\begin{aligned}
& \frac{d N^{0}}{d t}=\prod_{e} N^{+} n-N_{C N} N^{0} \underline{-} \times \sigma_{e N} v_{t h e}, N_{C N}=N_{C} \exp \& E_{N} / k T-
\end{aligned}
$$

Here $N_{V}=2.5 \times 10^{19} \mathrm{~cm}^{-3}$ and $N_{C}=1.9 \times 10^{19} \mathrm{~cm}^{-3}$ are the density of states in the valence and conduction band, $n$ and $p$ are the concentrations of free electrons and holes, $A l^{-}=N_{A l}-A l^{0}, N^{0}=N_{N}$ $-N^{+}$, where $N_{A l}$ is the total density of aluminum traps consisting of neutral $A l^{0}$ and ionized ones $A l^{-}$ $=\sim 3.5 \times 10^{14} \mathrm{~cm}^{-3}$ (by nitrogen compensation), $N_{N}=1.4 \times 10^{15} \mathrm{~cm}^{-3}$ is the nitrogen density, consisting of neutral $N^{0}$ and ionized $N^{+}$state. Aluminum and nitrogen activation energies of $E_{T}=$ $200 \mathrm{meV}$ and $E_{N}=60 \mathrm{meV}$ were used, respectively. Solution of Eq. (1) at quasi-equilibrium conditions (assuming that electron capture is faster than recombination) for photo-neutralized aluminum traps is $A l^{0}=\left(N^{*}+N_{A l}+\Delta N\right) / 2-\left[\left(N^{*}+N_{A l}+\Delta N\right)^{2} / 4-(\Delta N) \cdot N_{A l}\right]^{1 / 2}+n_{0} A l^{-} / P_{V A l}, N^{*}=$ $N_{V T} / g_{h}$ and for filled nitrogen as $N^{0}=N_{N} /\left(1+N_{C N} /\left[g_{e}\left(n_{0}+\Delta N\right)\right]\right) . g_{e}=2$ and $g_{h}=4$ are typical degeneracy factors for conduction and valence bands, respectively [34,35]. The relations at low excitations provide weakly filled $\mathrm{Al}$ traps of $A l^{0}=0.013 \times A l^{-}$and low concentration of neutral nitrogen $N^{0}=2.8 \times 10^{12} \mathrm{~cm}^{-3}$. Therefore, at low excitations the impurities are weakly neutralized and provide low PL signal, while with increase of excitation they are saturated with holes and electrons, when quasi-Fermi levels pass the $\mathrm{Al}$ and $\mathrm{N}$ trap levels. The fit according $P L \sim N^{0} A l^{0}$ relation is provided in Fig. $3 \mathrm{~b}$ and well describes obtained results. Notable is that aluminum concentration is much lower than $N^{*}$, thus the fit cannot provide its concentration.

It is known that $\mathrm{Al}$ impurities can reduce carrier lifetime [36] with $\left(N_{A l} / 5 \times 10^{18} \mathrm{~cm}^{-3}\right)^{1.2} \times 20$ ns relation. For our sample with $\mathrm{Al}$ density of $(3.5 \pm 0.5) \times 10^{14} \mathrm{~cm}^{-3}$ the relationship would provide 3 ms lifetime. For moderately Al doped $4 \times 10^{18} \mathrm{~cm}^{-3}$ sample, when $\mathrm{Al}$ is mostly in neutral form (this $\mathrm{Al}^{0}$ state can capture electrons providing $\mathrm{Al}$ related recombination process), lifetime is reduced by 20 ns [36], thus extrapolating to our Al concentration, we obtain Al related lifetime of $230 \mu \mathrm{s}$, which still strongly exceeds observed one. Moreover, lifetime of $2.6 \mathrm{~ms}$, observed in $2 \times 10^{17} \mathrm{~cm}^{-3}$ Al-doped SiC by MPCD [37] decreased with temperature increase due to Al activation to $120 \mu \mathrm{s}$ (note, that such large and reducing with temperature values can be due to $\mathrm{Al}^{0}$ activation in compensated samples [38]), which is opposite to our observations, indicating that temperature dependence of lifetime in our sample is governed by other kind of nonradiative defects which will be discussed in the next section. 
This is corrected version of manuscript with changes made according to the Reviewers' comments and submitted to Journal of Luminescence. The final version is available here:

https://www.sciencedirect.com/science/article/pii/S0022231318314339
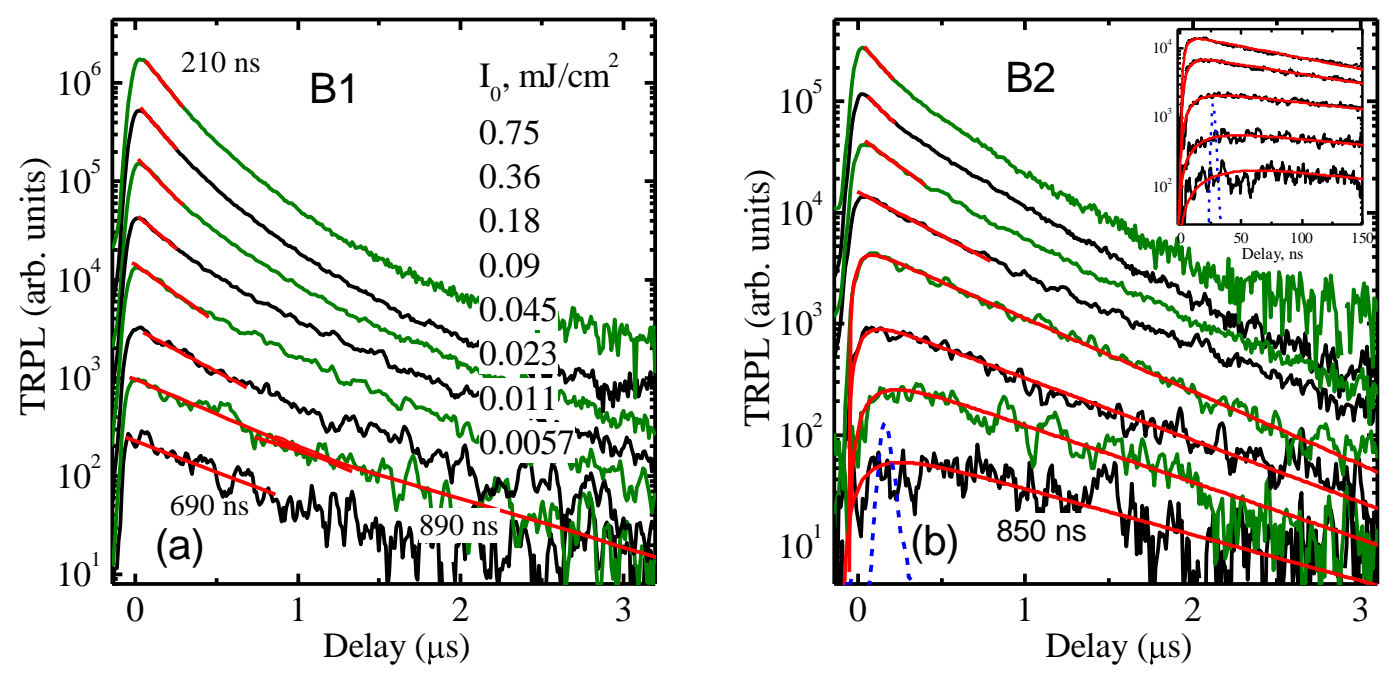

Fig. 4. TRPL decays for B1 (a) and B2 (b) PL bands measured in large temporal window, straight lines are exponential fits, while rising curves in (b) are numerical fits providing defect filling and emptying times; inset in (b) shows the initial parts of five upper curves measured in small temporal window. Note that response function (dashed curve) is much faster than the PL build-up time.

\subsection{Excitation and temperature dependences of recombination lifetimes}

The dependences of lifetime on excitations in different time intervals of DT or MPCD decay (Fig. 5) have shown gradually decreasing with excitation carrier lifetime. The latter effect is more pronounced at short delay times which is a consequence of density-dependent surface recombination rate $S(\Delta N)$. The latter effect was found more pronounced in a thin layer, as described in detail in [13]. The spatio-temporal carrier distribution after photoexcitation was calculated using a balance equation (2) [39]. This procedure allowed calculation of the excess carrier in-depth profile $\Delta N(z, t)$ in the epitaxial layer using standard boundary conditions [40] and taking into account carrier recombination in the bulk (by SRH lifetime $\tau_{S R H}$ ) and at the surface (total lifetime is descibed as $1 / \tau_{\text {tot }}=1 / \tau_{S}+1 / \tau_{\text {bulk }}$ ), as well as temperature and injection dependent ambipolar diffusion coefficient $D(T, \Delta N)$ in $\operatorname{SiC}[21,41]$ :

$$
\frac{\partial \Delta N(z, t)}{\partial t}=D \boldsymbol{\gamma}, \Delta N \frac{\partial^{2} \Delta N(z, t)}{\partial z^{2}}-\frac{\Delta N(z, t)}{\tau_{S R H} \boldsymbol{\gamma}, \Delta N}-C \backsim N \Delta N^{3}(z, t)+G(z, t) .
$$


This is corrected version of manuscript with changes made according to the Reviewers' comments and submitted to Journal of Luminescence. The final version is available here:

https://www.sciencedirect.com/science/article/pii/S0022231318314339

Here $G(z, t)=\left[\alpha(T) I_{0} / h v\right] \times \exp (-\alpha z)$ is the carrier generation rate [3], where $\alpha(T)$ dependence was taken from [17]. The solution of Eq. (2) is determined by applying standard boundary conditions: $\quad \Delta N \boldsymbol{Q} / d, t= \pm \frac{D(\Delta N)}{S(\Delta N)} \frac{\partial \Delta N \boldsymbol{Q} / d, t}{\partial z}$ where $S(\Delta N)$ is the fitted surface recombination rate on layer surfaces [3]. The dependence of surface recombination rate on carrier density was determined by fitting $\tau_{\text {tot }}$ in 30-130 ns delay intervals in the layer similarly as in [13], leading to $\tau_{\text {Sinit }}$ and consequently $S(\Delta N)=140 \times\left(1+2 \Delta N / n_{0}\right)^{1 / 2} \mathrm{~cm} / \mathrm{s}$, being 3 times lower than in a thin layer [13] due to better surface polishing conditions. In present work lower $S$ and larger $d$ favor to more precise determination of bulk lifetime.

The determined $S$ value provided $\tau_{S}$ at different excitations and thus bulk lifetime depencence on excitation was extracted; the latter was governed by $\tau_{S R H}$ lifetime (as impact of nonlinear recombination [21] is rather weak at low excitations). It was found, that bulk lifetime decreased from $4 \mu \mathrm{s}$ to $460 \mathrm{~ns}$ with excitation (Fig. 5, solid line), while the stabilised surface recombination time $\tau_{S s t a b}=d / 2 S+(d / \pi)^{2} / D$ [42] was found much longer (in range of $46 \mu$ s to $6 \mu$ s). Therefore, bulk carrier lifetime value in stabilised conditions after $3 \mu$ s (in 4300-4500 ns range, see Fig. 6) is presumably governed by SRH recombination. The modeling has shown that impact of Auger recombination (with coefficient of $C(\Delta N)=5 \times 10^{-31}+7 \times 10^{-13} / \Delta N \mathrm{~cm}^{6} / \mathrm{s}$ [21]) is negligible in excitation range up to $\Delta N<\approx 10^{17} \mathrm{~cm}^{-3}$, but above this density induces fast lifetime reduction (see kink in Fig. 5 and the dashed line).

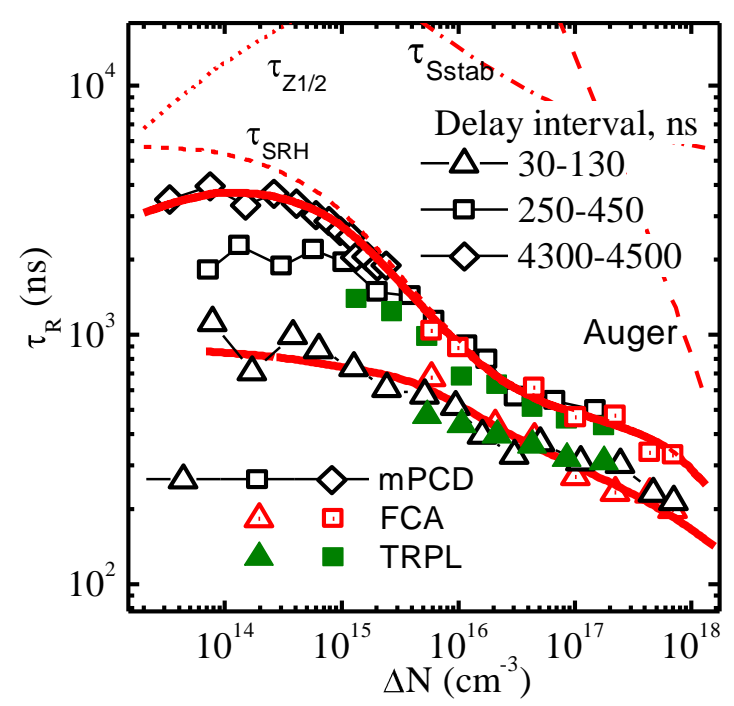

Fig. 5. Lifetime dependences on carrier density as determined by the three methods in different time intervals after photoexcitation pulse. Note, PL decay time was multiplied by factor of 2 as PL 
This is corrected version of manuscript with changes made according to the Reviewers' comments and submitted to Journal of Luminescence. The final version is available here:

https://www.sciencedirect.com/science/article/pii/S0022231318314339

$\Delta N^{2}$. Experimental points are scattered due to lifetime determination errors. Long-dashed, shortdashed, dotted and dash-dotted lines show Auger, SRH, $\mathrm{Z}_{1 / 2}$-related and stabilized surface lifetimes, respectively.

For analysis of $\tau_{\text {bulk }}(\Delta N)$ dependence, leading to lifetime decrease with excitation at $\Delta N>$ $10^{14} \mathrm{~cm}^{-3}$ (Fig. 1), the SRH recombination model [43] on traps was applied. We assumed that the hole traps govern the recombination process in the studied n-type sample. The shallow traps are assumed positively charged by capturing hole and after that electron capture occurs. Below, the model based calculations are given for the case when the initially filled trap concentration $N_{T}{ }^{+}$is much lower than the carrier density, i.e. $\Delta N+n_{0}>N_{T}^{+}$. The latter condition was verified by $N_{T}^{+}$ calculations (see values above using Eqs. (1) as an outcome of faster trap thermal emptying than filling as $N^{*} \gg N_{T}$ ). Thus, the $\mathrm{SRH}$ lifetime in our n-type $4 \mathrm{H}-\mathrm{SiC}$ layer can be expressed as follows [43]:

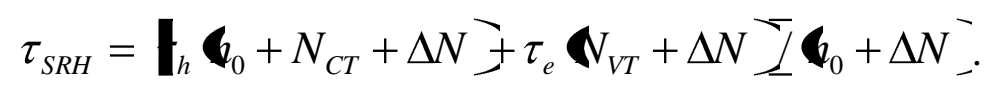

Here, $\tau_{e}$ and $\tau_{h}$ are electron and hole lifetimes, $n_{0}$ is the equilibrium electron concentration due to shallow nitrogen donors, $\left.N_{C T}=N_{C} \exp \boldsymbol{E}_{T}-E_{C}\right\rceil k T_{-}^{-}$is the electron concentration in conduction band when Fermi level is equal to defect level $E_{T}, N_{V T}=N_{V} \exp \left\langle E_{V}-E_{T}\right\rceil k T_{-}^{-}$is the hole concentration in the valence band when Fermi level is equal to $E_{T}$. $N_{C T}$ in latter would be negligibly small and thus was disregarded. Here, $\tau_{e}=1 / \mathbb{N}_{T} \sigma_{e} v_{t h e_{-}}, \tau_{h}=1 / \mathbb{N}_{T} \sigma_{h} v_{t h h}$, $v_{\text {th } e}=\sqrt{8 / \pi k T / m_{e d}{ }^{*}}$, and $v_{\text {th } h}=\sqrt{8 / \pi k T / m_{h d}{ }^{*}}$ are electron and hole lifetimes and thermal velocities, respectively; $\sigma_{e}$ and $\sigma_{h}$ are electron and hole capture cross sections to the trap, with $N_{T}$ density, respectively. Solid lines in Fig. 5 indicate $\tau_{\text {tot }}$ fits $\left(1 / \tau_{\text {tot }}=1 / \tau_{S R H}(\Delta N, T)+1 / \tau_{\text {Sstab }}(\Delta N, T)+\right.$ $1 / \tau_{Z 1 / 2}(\Delta N, T)$ for upper curve and $1 / \tau_{\text {tot }}=1 / \tau_{S R H}(\Delta N, T)+1 / \tau_{\text {Sinit }}(\Delta N, T)+1 / \tau_{Z 1 / 2}(\Delta N, T)$ for lower curve. Therein $\tau_{\text {Sstab }}(\Delta N, T)$ and $\tau_{\text {Sinit }}(\Delta N)$ are stabilised and initial surface lifetimes as determined by numerical simulation by Eqs. (2) in $4 \mu$ s and 30-130 ns intervals, respectively. The $\tau_{Z 1 / 2}(\Delta N, T)$ is $\mathrm{Z}_{1 / 2}$ defect recombination lifetime, calculated according model described in [1]. Using $4 \mathrm{H}-\mathrm{SiC} m_{e d}=$ $0.37 m_{0}, m_{h d}=1.0 m_{0}$, the thermal velocities $v_{\text {th }}=1.4 \times 10^{7} \mathrm{~cm} / \mathrm{s}$ and $v_{\text {th }}=0.8 \times 10^{7} \mathrm{~cm} / \mathrm{s}$ were calculated. The high injection lifetime at $300 \mathrm{~K}$ is $460 \mathrm{~ns}$. Using $N_{C}(T)=1.9 \times 10^{19}(T / 300)^{3 / 2} \mathrm{~cm}^{-3}$, $N_{V}(T)=2.5 \times 10^{19}(T / 300)^{3 / 2} \mathrm{~cm}^{-3}[44], n_{0}=10^{15} \mathrm{~cm}^{-3}$, and Eq. (3), we determine in Fig. $5 N_{V T} / n_{0}=$ 
This is corrected version of manuscript with changes made according to the Reviewers' comments and submitted to Journal of Luminescence. The final version is available here:

https://www.sciencedirect.com/science/article/pii/S0022231318314339

16, which leads to $E_{T}=190 \mathrm{meV}$, and finally $\tau_{h}=100 \mathrm{~ns}$ and $\tau_{e}=360 \mathrm{~ns}$. At low excitations traps are filled only partially with $N_{T}^{+} / N_{T}^{0}=n_{0} / N_{V T}=0.06$ factor, thus electron recombination is slowed down to $16 \times 360 \mathrm{~ns}=5.8 \mu$ s. Decrease of lifetime with excitation is due to increase of trap filling with holes and thus faster electron capture to them.

In Fig. 6, experimental data of lifetime temperature dependence (obtained from slow FCA decay tails at $\Delta N \sim 10^{16} \mathrm{~cm}^{-3}$ and almost stabilized profile conditions) are compared with carriercapture times by the shallow defect. Assuming $\sigma_{h} \sim T^{0}$ is temperature independent (neutral trap [45], similarly as for $\mathrm{Z}_{1 / 2}$ [1]), while the electron lifetime increases with temperature, $\sigma_{e} \sim T^{-2}$ (Coulombic trap [45]), we fitted the temperature dependence as shown by solid line in Fig. 6a. The dotted line indicates simulated by Eq. (3) bulk lifetime $\tau_{b u l k}$, while the dashed lines - the surface lifetime $\tau_{\text {Stab }}$, calculated using the determined $S$ value of $640 \mathrm{~cm} / \mathrm{s}$ (at $10^{16} \mathrm{~cm}^{-3}, 300 \mathrm{~K}$ and taking $S(T) \sim \exp \left(-\phi_{B} / k T\right)$, where $\phi_{B}=\sim 260 \mathrm{meV}$ [40] is the surface defect capture barrier). Also electron and hole lifetime temperature dependences are indicated by thin dashed lines. Deviation of the fit from data at $T>400 \mathrm{~K}$ could be due to partially unstabilized carrier profile (absorption coefficient strongly increases with temperature increase [17] while $D(T)$ decreases [21], thus carrier density profile stabilization time is much longer as $\left.\tau_{S s t a b}=d / 2 S+(d / \pi)^{2} / D\right)$. Overall in the fit we used the same $E_{T}=190 \mathrm{meV}$ trap and corresponding electron and hole lifetimes $\tau_{e}=360 \times(T / 300)^{3 / 2} \mathrm{~ns}$ and $\tau_{h}=100 \times(T / 300)^{-1 / 2} \mathrm{~ns}$, respectively; and temperature dependent $N_{V T}$.
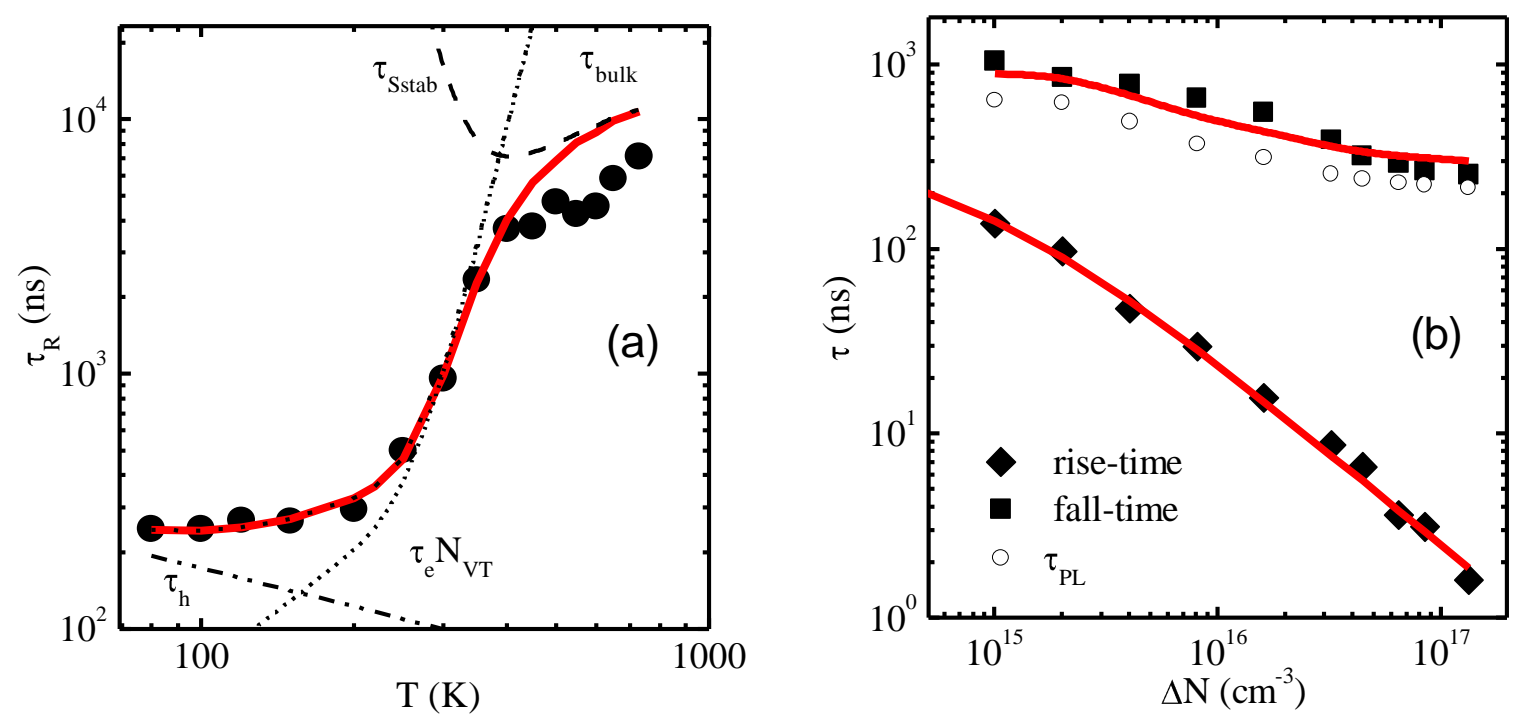

Fig. 6. Carrier lifetime temperature dependence at $\Delta N \sim 10^{16} \mathrm{~cm}^{-3} \gg N_{T}^{+}, n_{0}$ (a) and density dependent defect decay fitting parameters as determined from TRPL (b). Lines are numeric fits (see descriptions in the text). 
This is corrected version of manuscript with changes made according to the Reviewers' comments and submitted to Journal of Luminescence. The final version is available here:

https://www.sciencedirect.com/science/article/pii/S0022231318314339

Fig. $6 \mathrm{~b}$ presents PL rise-times, $\tau_{r}$, and fall-times, $\tau_{f}$ (i.e. defect filling and emptying times), as follows from fitting the TRPL kinetics (see Fig. 4b). The fall-time varies with excitation in the same interval as carrier lifetime in Fig. $4 \mathrm{~b}$; however, it has larger values than $\tau_{P L}$, which indicates for hole accumulation in $\mathrm{Al}^{-}$states. The fall-time of PL signal is described using instantaneous lifetime fit $\tau_{\text {fall }}=-x /(d x / d t)$, where $x=N_{T}^{+}(\Delta N) \times N^{0}(\Delta N) \sim P L_{\mathrm{B} 2} \sim \Delta N^{1.5}$ of band 2 and $\tau_{\text {fall }}=\tau_{R} / 1.5$. The latter fit is provided by the upper curve in Fig. 6b. However, DAP band B2 PL intensity is almost quadratic at low excitation (see Fig. 3b) which appears due to slow ionized aluminum filling with excited holes at low excitations, as their density partially decreases due to surface recombination and causes deviation from the numeric fit at low $\Delta N$. The $\mathrm{B} 2$ exhibited saturation attributable to saturation of nitrogen and aluminum traps at high excitation, as PL $\sim N^{0} A l^{0}$ (see latter fit in Fig. 3b, calculated by Eq. (1)).

The band B2 rise-time (defect filling time) is shortening with excitation and showing saturation at the highest carrier densities. The rise-time fit of defect band can be obtained by equation $P L(t)=N_{t 2}\left(1-\left(1-N_{t 1} / N_{t 2}\right) \times \exp \left(-t / \tau_{r}\right)\right) \times \exp \left(-t / \tau_{f}\right)$, where indices 1 and 2 correspond to the initial and final concentrations of filled nitrogen traps derived by Eqs. (1). The fits provided in Fig. $4 \mathrm{~b}$ were convoluted with response functions of $40 \mathrm{~ns}$ and $3 \mathrm{~ns}$ at FWHM in $5 \mu$ s and $200 \mathrm{~ns}$ windows, respectively. It was observed that rise-time decreases with excited carrier density as at higher carrier density defect filling is faster, and is provided by relation $\tau_{r}=1 / \Lambda N+n_{0} \bar{\sigma}_{e} v_{\text {th } e}$; Fitting of it in Fig. $6 \mathrm{~b}$ provides $\sigma_{e}=3.5 \times 10^{-16} \mathrm{~cm}^{2}$ for electron capture to $\mathrm{N}^{+}$center. For nitrogen electron capture cross section is $1.6 \times 10^{-13}$ and $4 \times 10^{-15}$ for 140 and $90 \mathrm{meV}$ species, respectively [46]. Taking into consideration lower cross section for $90 \mathrm{meV}$ nitrogen species (our species are shallower $(60 \mathrm{meV})$, thus can have lower cross section), the latter can be responsible for our risetime. Aluminum capture cross section for holes is much larger $(0.3-4.5) \times 10^{-12} \mathrm{~cm}^{2}$ [46]. In literature boron provides hole capture cross section of $(1.8-10) \times 10^{-14} \mathrm{~cm}^{2}$ and $\sim 300 \mathrm{meV}$ activation energy [41], thus it would not explain the observed slow risetime and DAP spectra.

Equation (3) predicts longer lifetime for less doped sample. This tendency of minority carrier lifetime decrease in 1400-400 ns range with $n_{0}$ increase from $10^{15}$ to $10^{16} \mathrm{~cm}^{-3}$ has been observed in [7] in $4 \mathrm{H}-\mathrm{SiC}$ with low $\mathrm{Z}_{1 / 2}$ concentration, $\sim 2 \times 10^{12} \mathrm{~cm}^{-3}$. It is notable, that lifetime decrease by order of magnitude with excitation increase in our studied bulk sample is in strong contrast with lifetime increase with excitation in bulk $4 \mathrm{H}-\mathrm{SiC}$, reported by [1]. In the latter samples, typical $\mathrm{Z}_{1 / 2}$ defects with $E_{a} \sim 0.6 \mathrm{eV}$ were dominant with $\sim 10^{13} \mathrm{~cm}^{-3}$ concentration. Thus we show that other defects dominate excitation dependent recombination rate in our studied sample. Our 
This is corrected version of manuscript with changes made according to the Reviewers' comments and submitted to Journal of Luminescence. The final version is available here:

https://www.sciencedirect.com/science/article/pii/S0022231318314339

approach provides $4.5 \mu \mathrm{s}$ minority carrier lifetime for $10^{15} \mathrm{~cm}^{-3}$ equilibrium electron density. In comparison, few times longer lifetime $(\sim 13 \mu \mathrm{s})$ has been observed in low doped $5-7 \times 10^{14} \mathrm{~cm}^{-3}$ samples with lower $Z_{1 / 2}$ densities $\left(<10^{12} \mathrm{~cm}^{-3}\right.$ ) [47], and even longer liftime (of $\sim 48 \mu \mathrm{s}$ ) in $235 \mu \mathrm{m}$ thick sample with $10^{14} \mathrm{~cm}^{-3}$ doping [5]. In such cases, $\mathrm{Z}_{1 / 2}$ are eliminated, but the impurity defects as titanium and other hole traps can govern the process of recombination.

Recently, Ti doped $5 \times 10^{14} \mathrm{~cm}^{-3}$ and $\mathrm{N}$ codoped $5 \times 10^{18} \mathrm{~cm}^{-3} 4 \mathrm{H}-\mathrm{SiC}$ provided minority carrier lifetime of $10 \mathrm{~ns}$ at RT [48]. According to equation (3), the high n-type doping gives conditions when Ti would be filled with electrons and lifetime would be limited by minority hole capture to $\mathrm{Ti}^{-}$. Electron and hole lifetime relations $\tau_{e}=360 \times(\mathrm{T} / 300)^{-1 / 2} \mathrm{~ns}$ and $\tau_{h}=100 \times(\mathrm{T} / 300)^{3 / 2}$ ns can be obtained using instead of hole an $170 \mathrm{meV}$ electron trap in Eq. (3). Ten times shorter hole lifetime at RT in our sample would provide $5 \times 10^{13} \mathrm{~cm}^{-3}$ concentration of Ti, and electron and hole capture cross sections of $\sigma_{e}=4 \times 10^{-15} \mathrm{~cm}^{2}$ and $\sigma_{h}=2.5 \times 10^{-14} \mathrm{~cm}^{2}$ at RT according $\tau_{e}=1 / N_{T} \sigma_{e} v_{\text {th } e_{-}}$and $\tau_{h}=1 / \mathbb{N}_{T} \sigma_{h} v_{\text {th } h_{-}}$relations, respectively. In literature similar $\sigma_{e}=3 \times 10^{-15}$ $\mathrm{cm}^{2}$ and activation energy $160 \mathrm{meV}$ for Ti on cubic site were reported [12,49]. The Ti concentration for high quality $4 \mathrm{H}-\mathrm{SiC}$ grown in $\mathrm{C}$ rich conditions is known to be in $(2-11) \times 10^{13} \mathrm{~cm}^{-3}$ range [12]). However, Ti concentration of $(0.5-5) \times 10^{12} \mathrm{~cm}^{-3}$ is typical for the herein studied $4 \mathrm{H}-\mathrm{SiC}$, grown by chemical vapor deposition [50]. In case $\mathrm{Ti}$ would dominate luminescence, an additional $90 \mathrm{meV}$ hole trap above valence band with low capture cross section should be present in PL spectra in order to explain slow risetime of DAP band.

The determined unknown shallow trap can be more plausibly ascribed to silicon vacancy defects as samples grown at carbon rich conditions lack in silicon atoms. The silicon vacancy $\mathrm{V}_{\mathrm{Si}}$ (0/-) at $E_{C}-930-985 \mathrm{meV}$ [51] has low anneal temperature of $\sim 800^{\circ} \mathrm{C}$ thus its formation during growth is questionable. However, in the presence of small amounts of intrinsic impurities as boron and nitrogen complexes of the silicon vacancies $\mathrm{V}_{\mathrm{Si}}$ are possible with them forming hole traps (+/0 charge states): with boron $\mathrm{B}_{\mathrm{C}} \mathrm{V}_{\mathrm{Si}}$ at $E_{V}+190 \mathrm{meV}$, with nitrogen $\mathrm{N}_{\mathrm{Si}} \mathrm{V}_{\mathrm{Si}}$ at $E_{V}+500 \mathrm{meV}$ [52]. Moreover, HS1 trap with large $2 \times 10^{-14} \mathrm{~cm}^{2}$ hole cross section attributed to silicon antisite $\mathrm{Si}_{\mathrm{C}}$ with position at $E_{V}+210-240 \mathrm{meV}$ activation energy was determined [2,12,53]. Latter would provide $6 \times 10^{13} \mathrm{~cm}^{-3}$ trap density in our sample, and $3.5 \times 10^{-15} \mathrm{~cm}^{2}$ its electron cross section. In comparison, boron hole capture cross section is similarly large $2 \times 10^{-14}-10^{-13} \mathrm{~cm}^{2}$ [46] and typical boron concentrations for herein studied samples are $(1-5) \times 10^{13} \mathrm{~cm}^{-3}[50]$, indicating $\mathrm{B}_{\mathrm{C}} \mathrm{V}_{\mathrm{Si}}$ trap is very plausible. Moreover, recently boron doping was used to reduce lifetime down to $30 \mathrm{~ns}$ [54]. Further investigations are needed for determining titanium impact and the shallow hole trap origin and its passivation route needed for $4 \mathrm{H}-\mathrm{SiC}$ lifetime enhancement at high excitations. 
This is corrected version of manuscript with changes made according to the Reviewers' comments and submitted to Journal of Luminescence. The final version is available here:

https://www.sciencedirect.com/science/article/pii/S0022231318314339

\section{Conclusions}

We conclude that combination of microwave photoconductivity, temperature- and time-resolved photoluminescence, and free carrier absorption techniques allowed to gain deeper insight into recombination pathways in $4 \mathrm{H}-\mathrm{SiC}$ with low $\mathrm{Z}_{1 / 2}$ defect density. The unusual bulk lifetime decrease with excitation from $4 \mu$ s to $460 \mathrm{~ns}$, observed in bulk $4 \mathrm{H}-\mathrm{SiC}$ crystal, was explained due to dominant impact of plausibly silicon vacancy related shallow hole traps at $E_{V}+0.19 \mathrm{eV}$. Surface and nonlinear recombination impacts were verified to be much weaker. Modeling of excitation dependent electron and hole capture by these traps provided electron (hole) lifetimes of $360 \mathrm{~ns}$ (100 ns) at $300 \mathrm{~K}$, correspondingly. The increase of lifetime with temperature was modeled with the same $190 \mathrm{meV}$ intrinsic trap, which governs electron lifetime increase with temperature due to its thermal activation and capture cross section reduction for electrons. Much lower shallow ( 60 $\mathrm{meV}$ ) nitrogen impurity capture cross section for electrons of $3.5 \times 10^{-16} \mathrm{~cm}^{2}$ was determined from $420 \mathrm{~nm}$ nitrogen-aluminum DAP band photoluminescence risetime becoming shorter with excitation.

Acknowledgements. We acknowledge collaborative links between Nagoya Institute of Technology and Vilnius university as well financial support by the Lithuania- Belarus joint research project No. 17-001 (F17LITG-009) founded by the Research Council of Lithuania and the Belarusian Republican Foundation for Fundamental Research.

\section{References}

[1] P. B. Klein, J. Appl. Phys. 103 (2008) 033702.

[2] P. B. Klein, A. Shrivastava, and T. S. Sudarshan, Phys. Status Solidi A 208 (2011) 2790-2795.

[3] P. Ščajev, V. Gudelis, K. Jarašiūnas and P. B. Klein, J. Appl. Phys. 108 (2010) 023705.

[4] S. Ichikawa, K. Kawahara, J. Suda and T. Kimoto, Appl. Phys. Express 5 (2012) 101301.

[5] T. Kimoto, K. Kawahara, B. Zippelius, E. Saito, J. Suda, Superlatt. Microstruct. 99 (2016) 151157.

[6] M. Kato, Y. Mori and M. Ichimura, Jpn. J. Appl. Phys. 44 (2015) 04 DP14.

[7] R. L. Myers-Ward, B. L. VanMil, K.-K. Lew, P. B. Klein, E. R. Glaser, J. D. Caldwell, M. A. Mastro, L. Wang, P. Zhao, C. R. Eddy, Jr., and D. K. Gaskill, J. Appl. Phys. 108 (2010) 054906.

[8] G. Feng, J. Suda, T. Kimoto, Appl. Phys. Lett. 92 (2008) 221906.

[9] Y. Ishikawa, M. Sudo, Y.-Z. Yao, Y. Sugawara, and M. Kato, J. Appl. Phys. 123 (2018). 225101. 
This is corrected version of manuscript with changes made according to the Reviewers' comments and submitted to Journal of Luminescence. The final version is available here:

https://www.sciencedirect.com/science/article/pii/S0022231318314339

[10] P. Ščajev, J. Hassan, K. Jarašiūnas, M. Kato, A. Henry and J. P. Bergman, J. Electron. Mater. 40 (2011) 394-399.

[11] B. Chen, H. Matsuhata, T. Sekiguchi, A. Kinoshita, K. Ichinoseki, and H. Okumura, Appl. Phys. Lett. 100 (2012) 132108.

[12] J. Zhang, L. Storasta, J. P. Bergman, N. T. Son, E. Janzen, J. Appl. Phys. 93 (2003) 4708.

[13] L. Subacius, K. Jarašiūnas, P. Ščajev and M. Kato, Meas. Sci. Technol. 26 (2015) 125014.

[14] P. Ščajev, S. Miasojedovas, A. Mekys, D. Kuciauskas, K. G. Lynn, S. K. Swain, and K.

Jarasiunas, J. Appl. Phys. 123 (2018) 025704.

[15] Y. Ichikawa, M. Ichimura, T. Kimoto, and M. Kato, ECS Journal of Solid State Science and Technology 7 (2018) Q127-Q130.

[16] T. Kimoto, K. Danno, and J. Suda, Phys. Stat. Solidi B 245 (2008) 1327-1336.

[17] P. Ščajev, M. Kato and K. Jarašiūnas, J. Phys. D: Appl. Phys. 44 (2011) 365402.

[18] P. B. Klein, R. Myers-Ward, K. -K. Lew, B. L. VanMil, C. R. Eddy, D. K. Gaskill Jr., A. Shrivastava and T. S. Sudarshan, J. Appl. Phys. 108 (2010) 033713.

[19] P. Ščajev, R. Aleksiejunas, S. Miasojedovas, S. Nargelas, M. Inoue, C. Qin, T. Matsushima, C. Adachi, and S. Juršènas, J. Phys. Chem. C 121 (2017) 21600-21609.

[20] M. V. Malashchonak, S. K. Poznyak, E. A. Streltsov, A. I. Kulak, O. V. Korolik and A. V. Mazanik, Beilstein J. Nanotechnol. 4 (2013) 255-261.

[21] P. Ščajev and K. Jarašiūnas, J. Phys. D: Appl. Phys. 46 (2013) 265304.

[22] P. B. Klein, B. V. Shanabrook, S. W. Huh, A. Y. Polyakov, M. Skowronski, J. J. Sumakeris, and M. J. O’Loughlin, Appl. Phys. Lett. 88 (2006) 052110.

[23] G. Feng, J. Suda, and T. Kimoto, Appl. Phys. Lett. 94 (2009) 091910.

[24] W. J. Choyke, Mater. Res. Bull. 4 (1969) 141-152.

[25] I. G. Ivanov, A. Henry, and E. Janzén, Phys. Rev. B 71 (2005) 241201(R).

[26] M. Ikeda and H. Matsunami, Phys. Stat. Solidi A 58 (1980) 657-663.

[27] G. Pensl, W. J. Choyke, Physica B 185 (1993) 264-283.

[28] J. Pernot, W. Zawadzki, S. Contreras, J. L. Robert, E. Neyret, and L. Di Cioccio, J. Appl. Phys. 90 (2001) 1869.

[29] I. G. Ivanov, B. Magnusson, and E. Janzen, Phys. Rev. B 67 (2003) 165211.

[30] S. G. Sridhara, L. L. Clemen, R. P. Devaty, and W. J. Choyke, D. J. Larkin, H. S. Kong, T. Troffer and G. Pensl, J. Appl. Phys. 83 (1998) 7909.

[31] M. Ikeda, H. Matsunami, and T. Tanaka, Phys. Rev. B 22 (1980) 2842-2854.

[32] P. Ščajev, M. Karaliūnas, E. Kuokštis and K. Jarašiūnas, J. Lumin. 134 (2013) 588-593. 
This is corrected version of manuscript with changes made according to the Reviewers' comments and submitted to Journal of Luminescence. The final version is available here:

https://www.sciencedirect.com/science/article/pii/S0022231318314339

[33] S. M. Sze, K. Ng, Physics of Semiconductor Devices, $3^{\text {rd }}$ Edition Wiley, 2006, ISBN: 978-0471-14323-9.

[34] D. K. Schroder, Semiconductor Material and Device Characterization Wiley-Interscience Publication, NY, 1990, ISBN: 0-471-51104-8.

[35] K. Seeger (2004). §3.2 Occupation probabilities of impurity levels, Semiconductor physics: An introduction, 9th ed. Springer, ISBN 3540219579.

[36] G. Liaugaudas, D. Dargis, P. Kwasnicki, R. Arvinte, M. Zielinski and K. Jarašiunas, J. Phys. D: Appl. Phys. 48 (2015) 025103.

[37] T. Okuda, H. Miyake, T. Kimoto, and J. Suda, Jap. J. Appl. Phys. 52 (2013) 010202.

[38] G. Liaugaudas, P. Ščajev and K. Jarašiūnas, Semicond. Sci. Technol. 29 (2014) 015004.

[39] M. Boulou and D. Bois, J. Appl. Phys. 48 (1977) 4713-4721.

[40] D. K. Schroder, Semiconductor Materials and Device Characterization, third edition John

Wiley \& Sons: New Jersey, 2006 Chap. 7.

[41] P. Grivickas, J. Linnros and V. Grivickas, J. Mater. Res. 16 (2001) 524-528.

[42] A. B. Sproul, J. Appl. Phys. 76 (1994) 2851-2854.

[43] W. Shockley and W. T. Read, Phys. Rev. 87 (1952) 835-842.

[44] C. Persson and U. Lindefelt, J. Appl. Phys. 82 (1997) 5496-5508.

[45] B. K. Ridley, Quantum processes in semiconductors Clarendon Press, Oxford, 1999.

[46] W. Kaindl, M. Lades, N. Kaminski, E. Niemann, and G. Wachutka, J. Electron. Mater. 28 (1999) 154-160.

[47] T. Kimoto, Y. Nanen, T. Hayashi, and J. Suda, Appl. Phys. Express 3 (2010) 121201.

[48] T. Miyazawa, T. Tawara and H. Tsuchida, Mater. Sci. Forum 897 (2017) 67-70.

[49] T. Dalibor, G. Pensl, N. Nordell and A. Schoner, Phys. Rev. B 55 (1997) 13618.

[50] T. Kimoto, Progress in Crystal Growth and Characterization of Materials 62 (2016) 329-351.

[51] M. V. S. Chandrashekhar, I. Chowdhury, P. Kaminski, R. Kozlowski, P. B. Klein and T.

Sudarshan, Appl. Phys. Express 5 (2012) 025502.

[52] E. Igumbor, O. Olaniyan, R. E. Mapasha, H. T. Danga, E. Omotoso and W. E. Meyer, J. Phys.: Condens. Matter 30 (2018) 185702.

[53] L. Torpo and M. Marlo, J. Phys.: Condens. Matter 13 (2001) 6203-6231.

[54] T. Miyazawa, T. Tawara, H. Tsuchida, Mater. Sci. Forum 897 (2017) 51-54. 